\title{
TRATAMENTO DO EFLUENTE GERADO NA PRODUÇÃO DE BIODIESEL POR PROCESSO OXIDATIVO AVANÇADO: REAGENTE DE FENTON
}

\author{
L. O. GONÇALVES ${ }^{1}$, R. B. P. MARCELINO ${ }^{1}$, A. L. EMRICHC ${ }^{1}$, C. AMORIM ${ }^{1}$, M. M. D. LEÃO ${ }^{1}$ \\ ${ }^{1}$ Universidade Federal de Minas Gerais, Departamento de Engenharia Sanitária e Ambiental \\ E-mail para contato: camila@ desa.ufmg.br
}

\begin{abstract}
RESUMO - O objetivo do trabalho é o estudo do processo oxidativo avançado reagente de Fenton para tratamento do efluente proveniente da produção de Biodiesel. A produção de biodiesel tem se destacado como uma das principais tecnologias para o tratamento e reciclagem de óleos usados, de resíduos alimentícios e domésticos, porém há uma produção de um efluente de difícil tratabilidade com características que inibem o tratamento biológico convencional, elevados teor de sólidos, de carga orgânica e uma baixa concentração de nitrogênio. Dessa forma o processo oxidativo avançado - reagente de Fenton tem como objetivo a oxidação da matéria orgânica em dióxido de carbono e água e/ou o aumento da biodegradabilidade dos compostos orgânicos persistentes até um valor compatível com o tratamento biológico. No tratamento do efluente é necessária uma acidificação inicial para quebra da emulsão, o que favorece a aplicação do Fenton, que se processa com maior eficiência em meio ácido. Com a acidificação há também remoção da carga orgânica e do teor de sólidos, diminuindo a quantidade de reagentes (ferro e peróxido de hidrogênio) necessários no processo Fenton. A otimização do tratamento pode significar redução de custos diante da redução na formação de lama após a neutralização. O processo combinado acidificação - Fenton alcançou uma eficiência de remoção de demanda química do oxigênio, superior a $70 \%$ para o efluente em estudo.
\end{abstract}

\section{INTRODUÇÃ̃}

Os combustíveis fósseis possuem grande potencial poluidor, fazendo com que seja crescente a busca por fonte alternativas e renováveis de energia. Os biocombustíveis oferecem benefícios, incluindo a redução de gases que provocam o efeito estufa, Reijnders (2006), uma alta disponibilidade a partir de fonte comum, características não poluentes, biodegradáveis e que contribuem para a sustentabilidade, Puppan (2002). O biodiesel tem diversas vantagens em comparação com diesel oriundo do petróleo, além de ser plenamente competitivo na maioria dos aspectos técnicos, Fuduka et al. (2001). A biomassa pode ser convertida em biocombustíveis via rota bioquímica e química, como etanol e o biodiesel.

Grande parte do biodiesel no Brasil é produzido a partir de semente leguminosas principalmente da soja, mas existe um crescimento considerável para gordura animal, como pode ser explicitado na Tabela 1. 
Tabela 1 - Matéria prima utilizada na produção de biodiesel (B100) no Brasil

\begin{tabular}{ccccccc}
\hline \multirow{2}{*}{ Matéria Prima $\left(\mathrm{m}^{3}\right)$} & 2006 & 2007 & 2008 & 2009 & 2010 & 2011 \\
\cline { 2 - 7 } & 65.764 & 353.233 & 967.326 & 1.250 .590 & 1.980 .346 & 2.171 .113 \\
Óleo de soja & 816 & 34.445 & 154.548 & 255.766 & 302.459 & 358.686 \\
Gordura animal $^{1}$ & 2.431 & 18.423 & 31.655 & 37.863 & 47.781 & 44.742 \\
Outros materiais Graxos $^{2}$ & 69.012 & 408.005 & 1.177 .638 & 1.614 .834 & 2.387 .639 & 2.672 .771 \\
Total & & &
\end{tabular}

${ }^{1-}$ Inclui gordura bovina, gordura de frango e gordura de porco.

${ }^{2-}$ Inclui óleo de palma, óleo de amendoim, óleo de fritura usado e outros materiais graxos.

Fonte: ANP, 2012.

Segundo Fuduka et al. (2001) para a obtenção do biodiesel, o óleo e a gordura vegetal ou animal são submetidos a uma reação química denominada transesterificação. Nessa reação, óleo e gordura vegetal ou animal reagem na presença de um catalisador, geralmente uma base, com um álcool, geralmente metanol, produzindo os correspondentes ésteres, da mistura de ácidos graxos que são encontrados no óleo e gordura vegetal ou animal e obtendo-se como subproduto da reação a glicerina. Diversos alcoóis podem ser utilizados nas reações de transesterificação de óleos vegetais, porém os melhores resultados são obtidos quando alcoóis de cadeia curta são utilizados, sendo assim, os alcoóis mais utilizados são o metanol e o etanol Moura (2010).

Normalmente para a produção de um produto há a geração de efluente. Para o biodiesel não é diferente, utilizando métodos tradicionais de lavagem e purificação dos ésteres, segundo Neves (2011) é necessário pelo menos 3 litros de água de lavagem por litro de biodiesel produzido. Efluentes provenientes da produção de biodiesel têm características de coloração branca opaca, presença de óleos e graxas, elevados teor de sólidos, de carga orgânica e uma baixa concentração de nitrogênio. Dessa forma tratamentos biológicos convencionais são ineficientes. Nesse contexto, os processos oxidativos avançados (POA) se inserem. Os POA são processos químicos que ocorrem na presença de fortes agentes oxidantes tais como radical hidroxila. Eles têm como objetivo a oxidação da matéria orgânica em dióxido de carbono e água e/ou a potencialização da biodegradação dos compostos orgânicos persistentes até um valor compatível ao tratamento biológico. Além do mais, é possível alcançar uma alta eficiência de destruição de poluentes orgânicos uma vez que os radicais hidroxilas $(\bullet \mathrm{OH})$ gerados têm um o alto poder reativo, Shun et al. (2007).

A forma pelo qual os radicais atacam moléculas orgânicas podem ser pela: adição de duplas ligações, abstração do átomo de hidrogênio e transferência direta de elétrons Amorim et al. (2009). Dentre a grande variedade de processos oxidativos avançados existentes é dado destaque ao processo de reagente de Fenton de fácil aplicabilidade e manuseio. Na reação de Fenton, a formação do radical - $\mathrm{OH}$ é catalisada pelo $\mathrm{Fe}^{2+} \mathrm{em} \mathrm{pH}$ ácido. $\mathrm{O}$ processo Fenton pode ser representado de forma resumida pela reação abaixo:

$$
\mathrm{Fe}^{+2}+\mathrm{H}_{2} \mathrm{O}_{2} \rightarrow \mathrm{Fe}^{+3}+\cdot \mathrm{OH}+\mathrm{OH}^{-}
$$

No tratamento do efluente da produção de biodiesel a acidificação se faz necessária antes de qualquer tratamento, para quebrar as moléculas de óleo emulsionado. O rompimento de uma emulsão pode ser facilitado através do uso de um coagulante / floculante ou alteração de pH. Nesse estudo 


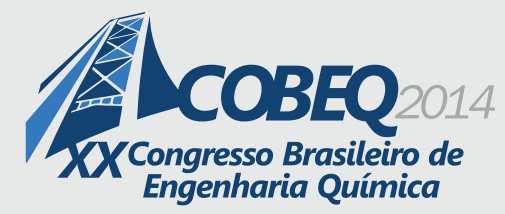

optamos pela alteração de $\mathrm{pH}$, dessa forma tornando-se economicamente viável a aplicação do Fenton, uma vez que já temos uma redução do pH 3,0 \pm 0,2 ideal para aplicação do tratamento do reagente de Fenton.

Com o pré-tratamento de acidificação também temos uma redução de carga orgânica, teor de sólidos, DQO e por conseqüência uma redução na formação de lama proveniente do Fenton, que ser forma após a neutralização. Independente do tipo de POA escolhido deve-se pensar na melhoria das condições operacionais objetivando a redução do custo do tratamento. Cada efluente tem suas particularidades e por isso deve haver uma escolha criteriosa da metodologia de tratamento.

Assim, o principal objetivo do trabalho foi avaliar o desempenho do reagente de Fenton no tratamento do efluente da produção de biodiesel.

\section{MATERIAIS E MÉTODOS}

Esse estudo foi realizado utilizando um efluente real obtido do processo de transesterificação alcalina de uma indústria, que utiliza como matéria-prima o óleo de fritura e o álcool etanol no processo produtivo. $\mathrm{O}$ efluente utilizado no estudo foi proveniente de uma amostragem única, pontual, sem nenhum tratamento preliminar.

Os estudos de oxidação avançada com reagente de Fenton foram realizados à temperatura ambiente, em reatores estáticos de bancada com agitação constante de $150 \mathrm{rpm}$, contendo $400 \mathrm{~mL}$ de efluente. Quantidades adequadas de sulfato ferroso heptahidratado $\left(\mathrm{FeSO}_{4} .7 \mathrm{H}_{2} \mathrm{O}\right)$ e de peróxido de hidrogênio $\left(\mathrm{H}_{2} \mathrm{O}_{2}\right.$ P.A) foram dosadas e considerou-se iniciado o processo oxidativo a partir da adição solução de peróxido de hidrogênio. A acidificação do efluente foi realizada através da solução $1 \mathrm{~N}$ de ácido sulfúrico $\left(\mathrm{H}_{2} \mathrm{SO}_{4}\right)$.

Alíquotas foram retiradas para determinação de demanda química de oxigênio (DQO) em tempos previamente determinados $(1,5,10,15,30,60,90,120$ e 180 minutos de reação). Simultaneamente, o teor de peróxido de hidrogênio residual dessas alíquotas foi determinado pelo método espectrofotométrico utilizando metavanadato de amônio segundo Nogueira et al. (2005). As amostras retiradas foram neutralizadas com $\mathrm{KOH} 5 \%$ e, em seguida, foi adicionada enzima catalase para reagir com o $\mathrm{H}_{2} \mathrm{O}_{2}$ residual para encerrar a reação de Fenton de acordo com procedimento descrito por Amorin et al. (2009). Todas as amostras analisadas foram previamente filtradas a vácuo utilizando membrana de fibra de vidro $45 \mathrm{~mm}$ (Sartorius) para remoção do ferro precipitado na etapa de neutralização.

O software STATISTICA ${ }^{\mathrm{R}}$ versão 8.0 foi utilizado para o planejamento do experimento, sendo realizado 4 ensaios. As reações de Fenton foram realizadas utilizando um planejamento fatorial $2^{2}$ podendo ser encontrado na Tabela 2. Obtendo diferentes concentrações para $\left[\mathrm{H}_{2} \mathrm{O}_{2}\right]$ e o $\left[\mathrm{Fe}^{2+}\right]$ a um $\mathrm{pH}(3,0+0,2)$.

Tabela 2 - Planejamento factorial dos exoerimentos com reagente de Fenton

\begin{tabular}{cccc}
\hline Ensaios & {$\left[\mathrm{Fe}^{2+}\right] \mathrm{mg} \cdot \mathrm{L}^{-1}$} & {$\left[\mathrm{H}_{2} \mathrm{O}_{2}\right] \mathrm{mg} \cdot \mathrm{L}^{-1}$} & $\mathrm{pH}$ \\
\hline 1 & 200 & 1.000 & $3,0 \pm 0,2$ \\
2 & 500 & 1.000 & $3,0 \pm 0,2$ \\
4 & 500 & 3.000 & $3,0 \pm 0,2$ \\
3 & 200 & 3.000 & $3,0 \pm 0,2$ \\
\hline
\end{tabular}


O teste de Zahn-Wellens indica consumo de matéria orgânica do efluente pelos microorganismos, quando há redução de DQO ou COT. Ensaios de biodegradabilidade foram realizados através do teste de Zahn-Wellens OCDE (1995), em que o efluente de biodiesel, lodo biológico de uma ETE Municipal e nutrientes minerais $\left(\mathrm{KH}_{2} \mathrm{PO}_{4}, \mathrm{~K}_{2} \mathrm{HPO}_{4}, \mathrm{Na}_{2} \mathrm{HPO} 4, \mathrm{NH}_{4} \mathrm{Cl}, \mathrm{CaCl}_{2}\right.$, $\mathrm{MgSO}_{4}$ e $\mathrm{FeCl}_{3}$ ) foram colocados juntos em um reator de 2 litros. Foi feito um reator controle utilizando substrato biológico (glicose) com nutrientes, realizado sob as mesmas condições do teste. A mistura foi agitada e arejada no escuro durante 28 dias. O processo de biodegradação foi monitorado por determinação de COT.

\section{RESULTADOS E DISCUSÃO}

\subsection{Teste com Reagente de Fenton}

Com o pré-tratamento de acidificação foi obtida uma considerável redução de carga orgânica e do teor de sólidos e DQO conforme mostra a Figura 1 e por conseqüência uma redução na formação de lama proveniente do Fenton, que se forma após a neutralização. Essa redução está associada à quebra da emulsão oleosa presente no efluente separando as fases de óleo livre, facilmente removido por gravimetria, e de água.

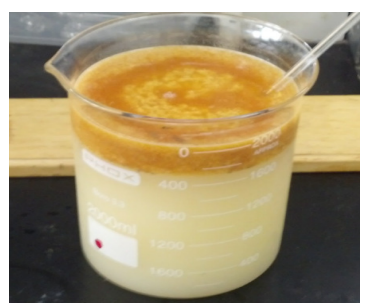

Figura 1 - Efluente do Biodiesel após acidificação.

O resultado da caracterização pode ser encontrado na Tabela 3 onde temos a caracterização do efluente bruto antes e após a sua acidificação, evidenciando a redução dos parâmetros citados acima.

Tabela 3 - Caracterização do efluente de biodiesel

\begin{tabular}{ccc}
\hline Parâmetros analisados & Efluente Bruto & Após acidificação \\
\hline DQO $\left(\mathrm{mgO}_{2} / \mathrm{L}\right)$ & 480.982 & 153.834 \\
$\mathrm{COT}(\mathrm{mg} / \mathrm{L})$ & 125.000 & 5.400 \\
$\mathrm{pH}$ & 8,7 & $3,0 \pm 0,2$ \\
Turbidez NTU & 249.000 & 120 \\
Cor $(\mathrm{mg} / \mathrm{L})$ & 488.300 & 430 \\
\hline
\end{tabular}

A Figura 2 apresenta o gráfico de superfície de resposta da remoção de DQO ao final de 180 minutos de reação de Fenton em função das variáveis das concentrações de ferro e peróxido obtidos no planejamento fatorial. De acordo com a Figura 2, uma região abrangendo concentrações de ferro inferiores a $500 \mathrm{mg} . \mathrm{L}-1$ e de peróxido inferiores ou igual a $3.000 \mathrm{mg} . \mathrm{L}^{-1}$ pode resultar em elevadas remoções. De maneira adicional, podemos indicar que razões $\left[\mathrm{Fe}^{2+}\right]:\left[\mathrm{H}_{2} \mathrm{O}_{2}\right]$ de 1:6 ou inferiores proporcionam remoções significativas pelo reagente de Fenton. A eficiência do Reagente de Fenton 
pode variar dependendo das condições experimentais.

A Figura 3 mostra o perfil do peróxido de hidrogênio consumido para cada configuração proposta. Nota-se que a cinética de consumo de peróxido de hidrogênio está relacionada à quantidade de ferro utilizado. Como pode ser observado, quando temos a menor concentração de peróxido, e maior a concentração de ferro maior a velocidade de reação, de forma esperada esse efeito contrário e visto para uma concentração maior de peróxido e menor concentração de ferro menor a velocidade de reação.

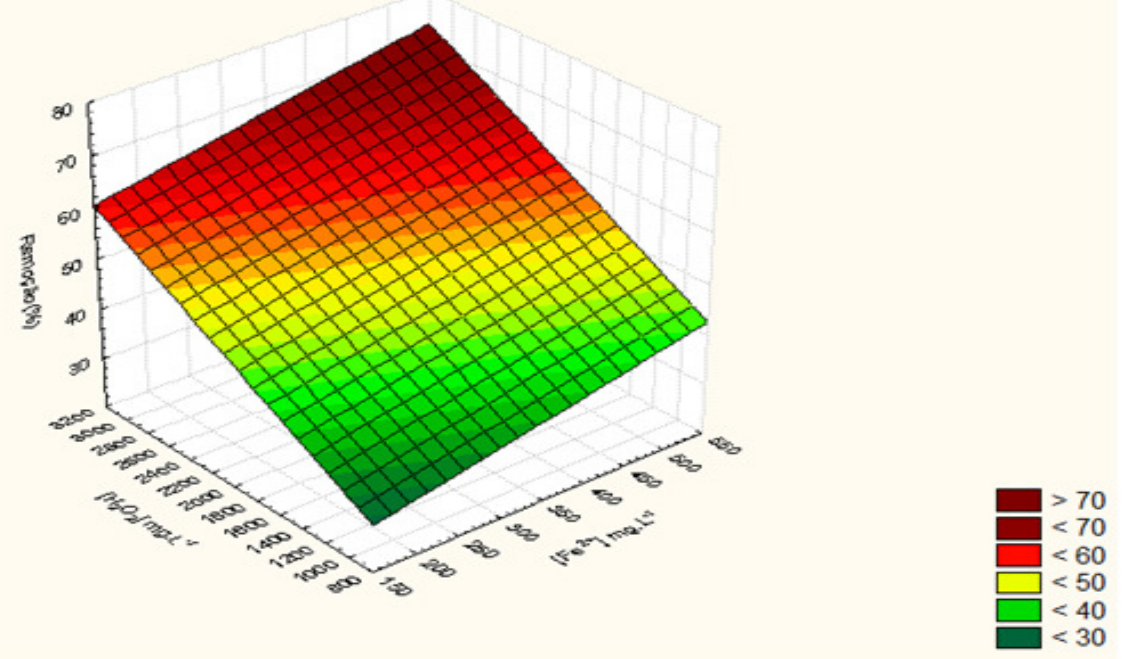

Figura 2 - Gráfico de superfície de resposta da remoção de DQO.

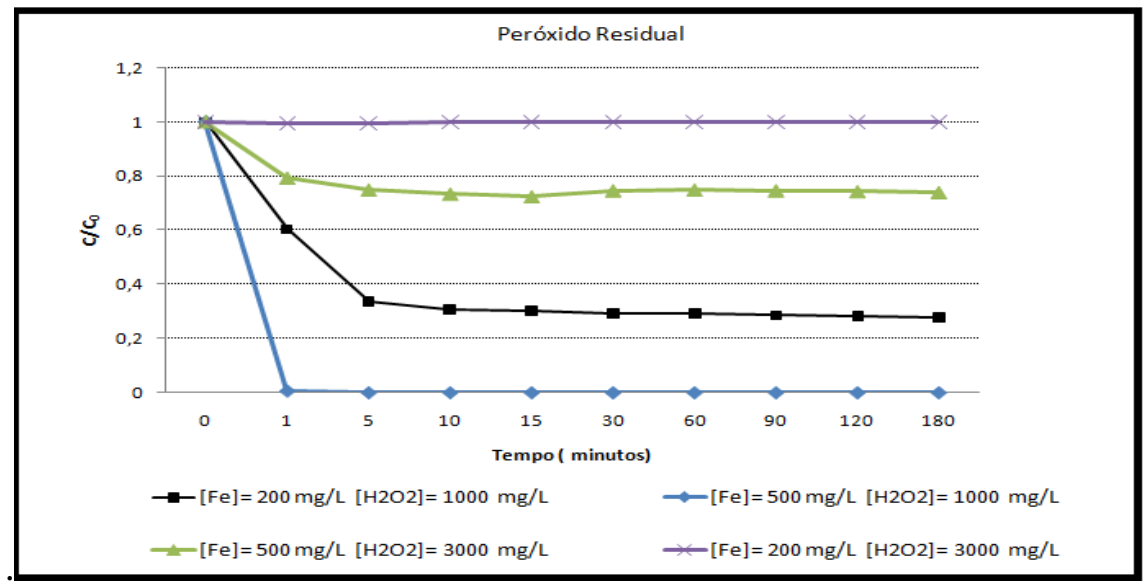

Figura 3 - Cinética do consumo de peróxido de hidrogênio.

A melhor relação entre as razões molares de ferro e peróxido respectivamente foi de 1:6, onde há consumo de peróxido e uma eficiência de remoção de $77 \%$ de DQO. Esse teor de peróxido de hidrogênio residual dessas alíquotas foi determinado pelo método espectrofotométrico utilizando metavanadato de amônio (NOGUEIRA et al., 2005). 
Tabela 4 - Razão molar do Ferro e peróxido

\begin{tabular}{|c|c|c|c|}
\hline$\left[\mathrm{Fe}^{2+}\right] \mathrm{mg} . \mathrm{L}^{-1}$ & {$\left[\mathrm{H}_{2} \mathrm{O}_{2}\right] \mathrm{mg} \cdot \mathrm{L}^{-1}$} & Eficiência de remoção de DQO(\%) & Razão Molar [Fe]:[ $\left.\mathrm{H}_{2} \mathrm{O}_{2}\right]$ \\
\hline 200 & 1000 & 41 & $1: 5$ \\
\hline 500 & 1000 & 36 & $1: 2$ \\
\hline 200 & 3000 & 52 & $1: 15$ \\
\hline 500 & 3000 & 77 & $1: 6$ \\
\hline
\end{tabular}

Os ensaios demonstraram que o reagente de Fenton é eficiente para aplicação específica no tratamento do efluente de biodiesel, comparada com literatura sugerida onde Mitre et al. (2012), obteve remoções na ordem de $80 \%$ de DQO, mas trabalhando com misturas de diesel e biodiesel. A Figura 5 mostra a sequiência do nosso estudo combinando acidificação + reagente de Fenton.

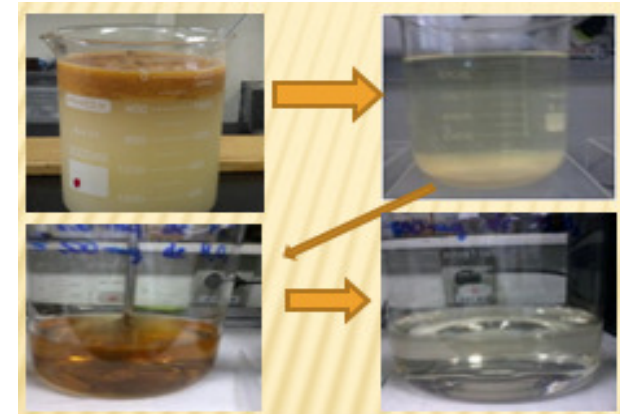

Figura 5- Efluente após o tratamento.

\subsection{Teste de Biodegradabilidade}

A biodegradabilidade do efluente de biodiesel é representada por uma remoção de COT mostrada na Figura 5. Os resultados demonstram que, após 28 dias, 96\% de redução de COT foi obtido. Apesar da alta eficiência de remoção de matéria orgânica, para biodegradabilidade é necessário longos períodos e o efluente tratado biologicamente ainda apresentaram altos valores de COT (6.500 mg L $\left.\mathrm{m}^{-1}\right)$ e DQO (24.600 mg L $\mathrm{m}^{-1}$ ). Isto indica que a aplicação de outros processos de tratamento ou de um tratamento biológico combinado com um processo de oxidação avançada faz-se necessário.

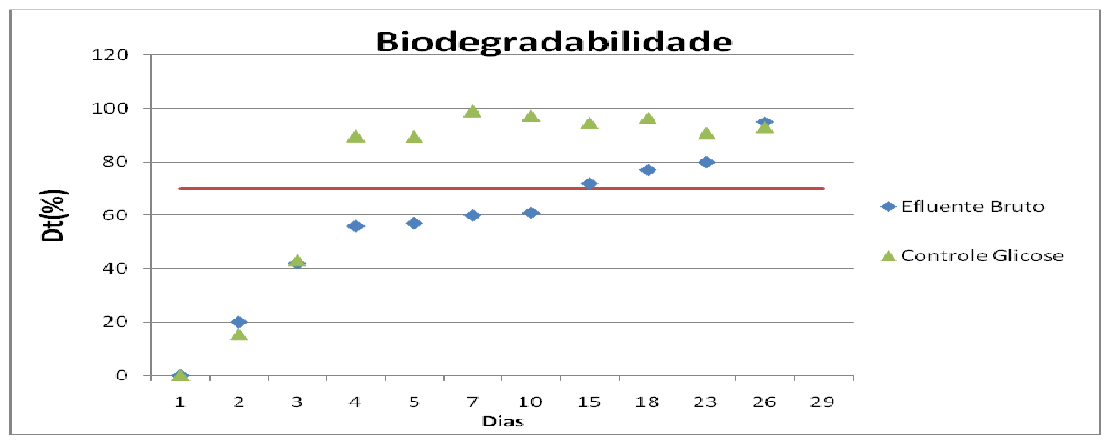

Figura 5 - Teste de biodegradabilidade Zahn-Wellens. 
Como indicado no protocolo o monitoramento do teste foi realizado por análise de COT para a realização do cálculo de Percentual de Biodegradação (Dt \%) segundo a equação 1:

$$
D_{t}=\left[1-\frac{C_{t}-C_{B}}{C_{A}-C_{B A}}\right] \times 100
$$

Equação (1)

Onde o CA e CBA correspondem ao COT $\left(\mathrm{mg} \mathrm{L}^{-1}\right)$ na amostra e no ensaio em branco, 3 horas após o início do ensaio, o Ct e CB correspondem ao COT $\left(\mathrm{mg} \mathrm{L}^{-1}\right)$ na amostra e no branco, medida na amostragem de tempo t. Segundo o método as amostras podem ser consideradas biodegradáveis quando o Percentual de Biodegradação atinge $70 \%$ é alcançado em até 14 dias de experimento. Esse resultado era esperado, conforme descrito na literatura, Suehara et al. (2005) observaram a biodegradabilidade no efluente de biodiesel diluído ao contrário do efluente bruto, onde não foi observado biodegradabilidade. Analisando os dados apresentados na Figura 5, verificou-se, que houve uma fase de adaptação dos micro-organismos nos quatro primeiros dias, com baixa biodegradabilidade.

\section{CONCLUSÃO}

Com esse estudo pode-se comprovar que a DQO presente no efluente da produção de biodiesel pode ser removido através do reagente de Fenton com eficiência superior a 70\%.Como está descrito na literatura, o valor de $\mathrm{pH}$ ótimo para a reação de Fenton é de 3,0 $\pm 0,2$, assim, a acidificação do efluente como tratamento preliminar requerido para a quebra da emulsão proporciona um efluente com valor de $\mathrm{pH}$ próximo ao tratamento de reagente de Fenton, reduzindo assim custos adicionais para acidificação do efluente , uma vez que o mesmo já foi acidificado para remoção de óleos e graxas.

Verificando através da perspectiva do custo de neutralização esse, está relacionado com o prétratamento de acidificação para retirada de óleos e graxas, pois a neutralização ocorreria mesmo que o reagente de Fenton não fosse aplicado para atender ao padrão de lançamento.

Verificando por essa ótica o custo do tratamento utilizando Fenton ficaria vinculado principalmente aos custos de aquisição dos reagentes de ferro e peróxido de hidrogênio, dessa forma, uma avaliação econômica deve ser realizada envolvendo os custos operacionais do processo visando à escolha do melhor tratamento para cada situação e condições ótimas operacionais.

O efluente em questão precisa de um processo combinado devido a sua baixa taxa de biodegradabilidade necessitado acima de 14 dias para atingir o percentual de $70 \%$ para ser considerado biodegradável.

\section{AGRADECIMENTOS}

Os autores agradecem à FAPEMIG e ao CNPQ pelo auxilio e financiamento da pesquisa. 


\section{REFERÊNCIAS}

Agência Nacional do Petróleo, Gás Natural e Biocombustível. Disponível em: <http://www.anp.gov.br/Downloads/69915.pdf> Acesso realizado em 06/set >

AMORIM, C.C., LEÃO M. M. D; MOREIRA R. F. P. M. Comparação entre diferentes processos oxidativos avançados para degradação de corante azo. Engenharia Sanitária e Ambiental, v. 14, p. 543-550, 2009.

FUKUDA, H.; KONDO, A; NODA, H. Biodiesel fuel production by transesterification of oils. $J$. Biosci Bioeng, v. 92, p. 405-416, 2001.

MITRE, T. K.; LEÃO, M. M. D.; ALVARENGA, M. C. N. Tratamento de águas contaminadas por diesel/biodiesel utilizando processo Fenton. Eng. Sanit. Ambiental. v.17, p. 129-136, 2012.

MOURA, B. S. Transesterificação Alcalina de Óleos Vegetais para Produção de Biodiesel: Avaliação Técnica e Econômica. 2010. 146. Dissertação - Pós-Graduação em Engenharia Química, Universidade Federal Rural do Rio De Janeiro. 2010.

NEVES, T. A. Tratamento físico-químico dos efluentes líquidos da produção de biodiesel metílico em regime de funcionamento contínuo e batelada. 2011. 103. Dissertação - PósGraduação em Engenharia de Edificações e Ambiental, Universidade Federal de Mato Grosso. 2011.

NOGUEIRA, R.F.P; OLIVEIRA, M.C.; PATERLINI, W.C. Simple and fast spectrophotometrtic determination of $\mathrm{H} 2 \mathrm{O} 2$ in photo-Fenton reactions using metavanadate, Talanta, v. 66, p. 86-91, 2005.

OECD. Detailed review paper on biodegradability testing environment monograph. 1995.

REIJNDERS, L. Conditions for the sustainability of biomass based fuel use. Energy Policy. v. 34, p.863-876, 2006.

PUPPAN, D. Environmental evaluation of biofuels. Periodica Polytechnica, v.10, p. 95-116, 2002.

SUEHARA, K.-I. et al. Biological treatment of wastewater discharged from biodiesel fuel production plant with alkali-catalyzed transesterification. Journal of Bioscience and Bioengineering, v. 100, n. 4, p. 437-442, 2005.

SUN, J. H.; SUN, S. P.; FAN, M. H.; GUO, H. Q.; QIAO, L. P.; SUN, R. X. A kinetic study on the degradation of $p$-nitroaniline by Fenton oxidation process. Journal of Hazardous Materials, v.148, p. 172-177, 2007. 